WellBeing International

WBI Studies Repository

\title{
$3-2011$
}

\section{Three Rs Approaches in the Production and Quality Control of Fish Vaccines}

\author{
Paul J. Midtlyng \\ Center for Veterinary Biologics \\ Coenraad F.M. Hendriksen \\ Netherlands Vaccine Institute \\ Elisabeth Balks \\ Paul-Ehrlich-Institut \\ Lukas Bruckner \\ Institut für Viruskrankheiten und Immunprophylaxe \\ Lawrence Elsken \\ Center for Veterinary Biologics
}

See next page for additional authors

Follow this and additional works at: https://www.wellbeingintlstudiesrepository.org/acwp_vsm

Part of the Animal Studies Commons, Other Animal Sciences Commons, and the Veterinary

Toxicology and Pharmacology Commons

\section{Recommended Citation}

Midtlyng, P. J., Hendriksen, C., Balks, E., Bruckner, L., Elsken, L., Evensen, Ø., ... \& Sneddon, L. U. (2011).

Three Rs approaches in the production and quality control of fish vaccines. Biologicals, 39(2), 117-128.

This material is brought to you for free and open access by WellBeing International. It has been accepted for inclusion by an authorized administrator of the WBI Studies Repository. For more information, please contact wbisr-info@wellbeingintl.org.

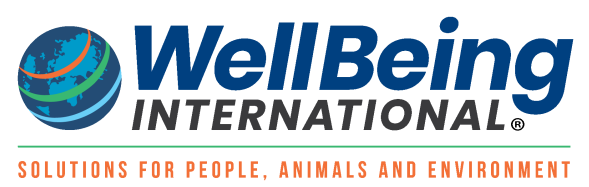




\section{Authors}

Paul J. Midtlyng, Coenraad F.M. Hendriksen, Elisabeth Balks, Lukas Bruckner, Lawrence Elsken, Øystein Evensen, Kjetil Fyrand, Allison Guy, Marlies Halder, Penny Hawkins, Gunn Kisen, Anne Berit Romstad, Kira Salonius, Patrick Smith, and Lynne U. Sneddon 


\title{
Three Rs Approaches in the Production and Quality Control of Fish Vaccines (Meeting Report)
}

\author{
Paul J. Midtlyng ${ }^{1,6}$, Coenraad Hendriksen ${ }^{2}$, Elisabeth Balks ${ }^{3}$, Lukas Bruckner ${ }^{4}$, Lawrence \\ Elsken $^{5}$, Øystein Evensen ${ }^{6}$, Kjetil Fyrand ${ }^{7}$, Allison Guy ${ }^{8}$, Marlies Halder ${ }^{9}$, Penny Hawkins ${ }^{10}$, \\ Gunn Kisen ${ }^{11}$, Anne Berit Romstad ${ }^{12}$, Kira Salonius ${ }^{13}$, Patrick Smith ${ }^{14}$, Lynne U. Sneddon ${ }^{15}$ \\ 1 VESO \\ 2 Netherlands Vaccine Institute \\ 3 Paul-Ehrlich-Institut \\ 4 Institut für Viruskrankheiten und Immunprophylaxe \\ 5 Center for Veterinary Biologics \\ Norwegian School of Veterinary Science \\ PHARMAQ \\ Canadian Council on Animal Care \\ Joint Research Centre European Commission \\ ${ }_{10}$ Royal Society for the Prevention of Cruelty to Animals \\ ${ }^{11}$ Norwegian Medicines Agency \\ ${ }^{12}$ Nord-Trondelag University College \\ ${ }^{13}$ Novartis Animal Health Canada Inc. \\ ${ }^{14}$ Schering Plough Animal Health \\ ${ }^{15}$ University of Liverpool
}

\section{KEYWORDS}

fish vaccines, licensing, quality control, efficacy, safety, potency, replacement, reduction, refinement

\section{ABSTRACT}

The workshop on Three Rs Approaches in the Production and Quality Control of Fish Vaccines aimed a) to identify animal tests currently stipulated for the production and quality control of fish vaccines and to highlight animal welfare concerns associated with these tests; b) to identify viable options to replace, reduce, and refine animal use for fish vaccine testing; and c) to discuss the way forward and set out how the Three Rs may be implemented without jeopardizing the quality of the vaccines. The workshop participants -- experts from academia, regulatory authorities, a scientific animal welfare organization, and the fish vaccine industry -- agreed that efforts should be undertaken to replace the vaccination challenge batch potency testing with tests based on antigen quantification or antibody response tests. Regulatory requirements of questionable scientific value and relevance for the quality of fish vaccines, such as the re-testing of batches produced outside Europe, or the double-dose batch safety test, should be reconsidered. As an immediate measure the design of the current animal tests should be evaluated and modified in the light of refinement and reduction, for example, the number of unprotected control fish in vaccination-challenge tests should be reduced to the minimum. 


\section{Introduction}

The European Centre for the Validation of Alternative Methods (ECVAM) organized a workshop on Three Rs Approaches in the Production and Quality Control of Fish Vaccines. The workshop took place on 29 e31 January 2008 in Ispra, Italy. The main objectives of the workshop were a) to identify animal tests currently stipulated for the production and quality control of fish vaccines and to highlight animal welfare concerns associated with these tests; b) to identify viable options to reduce, refine and replace animal use for fish vaccine testing; and c) to discuss the way forward and set out how replacement, reduction and refinement (Three Rs, [1]) may be implemented more actively.

Driven by remarkable successes in disease prevention and control, there is a significant increase in vaccination against bacterial and viral infections in European aquaculture, and a corresponding increase in vaccine supply to European markets. According to current regulatory guidelines, in vivo methods are still dominating the evaluation of current and new vaccines for fish. Consequently, there is an increase in the number of fish used during the development, licensing and quality control of fish vaccines. In Norway, which is the leading producer of farmed salmonids and the major user of fish vaccines in Europe, this has led to fish outnumbering all other animal species used for biomedical research [2]. Furthermore, regulatory requirements stipulate immunization challenge tests to demonstrate the efficacy of fish vaccines, and such tests are also recommended to confirm potency before batch release. Because unprotected fish will develop disease and illness during such tests, these procedures are of greater concern with respect to animal welfare than those which do not involve experimental challenge (e.g. serological tests). The potential for adverse reactions, and for test procedures to cause pain, suffering and distress, applies to fish in the same way as any other species.

In this context, experts from academia, regulatory authorities, a scientific animal welfare organization and the fish vaccine industry participated in the workshop, contributing unique scientific data, their personal experience, scientific skills and judgment. At the time of writing, several essential aspects relevant to animal welfare in fish are scarcely researched or may be subject to divergent interpretation (e.g. the nature of pain sensation and cognition in fish). However, the participants agreed on two overall goals. The first was to focus on how replacement, reduction and refinement of animal tests [1] could be further implemented particularly regarding procedures perceived as the "most severe". The second was to explore how animal tests of questionable relevance could be avoided in the production and quality control of fish vaccines.

Before presenting the outcome of the workshop, background information is provided on relevant topics such as the immune system of fish, the capacity of fish to experience pain, suffering and distress, and current fish vaccine testing requirements. Each of these has implications for the importance of and/or ability to implement the Three Rs concept in the quality control of fish vaccines.

\subsection{The immune system of fish}

The most prominent macroscopic differences between mammalian and teleost immune systems are the location and distribution of relevant cells, tissues and organs, especially the sites for hematopoiesis. The obvious differences are that fish lack bone marrow, lymph nodes and Peyer's patches, and instead the kidney is a major lymphoid organ in the teleosts in addition to the thymus, spleen and mucosa-associated lymphoid tissues $[3,4]$. Primary lymphoid organs seem to be the thymus (T-cells) and kidney (B-cells) [5]. The foremost part of the kidney shows morphological similarities with the bone marrow in higher vertebrates [6] but it also serves as a secondary lymphoid organ, and together with the spleen can be viewed as a lymph node analog important in the induction and elaboration of immune responses [7]. It appears that four out of the five classical inflammatory signs may be present in fish; swelling (tumor), 
redness (rubor), pain (dolor), and loss of function (functio laesa) but being poikilothermic animals, heat (calor) is not obviously associated with inflammation in fish. Mast-like cells (eosinophilic granule cells) are present but lack histamine in their granules [8], however, they degranulate following intraperitoneal injection with various phlogistic agents including mast cell degranulation agent 48/80 [9] suggesting that they induce vascular changes. The humoral components of the innate immune system of fish are numerous, with different functions and include growth inhibitors, various lytic enzymes, components of the complement pathway, agglutinins and precipitins, natural antibodies, cytokines, chemokines and antibacterial peptides. An overview of the innate immune system of fish can be found in $[4,10]$. The current status of the adaptive immune system in fish has been recently reviewed [3]. The effector cells of this system are two types of lymphocytes, B-cells that play the effector role in the humoral immune response, whereas T-cells are intimately involved in cell-mediated immune responses. Alongside with the lymphocytes is the presence of antigen presenting MHC class I and class II molecules however in all teleosts studied so far including salmonids the MHC class I and II regions are unlinked [11].

The availability of well-characterized B cell-specific anti-IgM monoclonal antibodies (mabs) for many fish species has facilitated more researchon B-cells compared to T-cells. Compared to mammals, fish B cell responses differs in many aspects. They do not show an Ig class switch, and the only classes detected so far is a tetrameric homolog of IgM as predominant serum immunoglobulin and two other types, IgD and IgT [12-14]. In contrast to mammalians, B-cells from teleost fish show potent in vitro and in vivo phagocytic activities [15]. Bonyfish respond however poorly to immunization compared to mammals as they produce antibodies with lower affinity for the antigen as affinity maturation has not been detected in fish [16].

Detailed functional studies of piscine T-cells in major aquaculture species are currently hindered due to lack of antibodies for detection, but their presence can be concluded from functional studies and expression of TCR, CD8 and CD4 genes [3]. Studies on cell-mediated cytotoxicity (CMC) activity in fish are also hindered due to highly polymorphic MHC molecules [17] although experimental studies have documented MHC-I restricted killing of virus infected target cells [18] and similar findings were reported earlier in rainbow trout [19]. To what extent the $T$ helper cell subdivision exists in fish is not known but some of the cytokines reflecting both $\mathrm{Th}_{1}$ and $\mathrm{Th}_{2}$ responses have been found in salmonids, zebrafish, and pufferfish.

The second key feature of the adaptive immune response is memory and albeit indications of the existence of an immunological memory in fish [20], however, it is not definitively proven.

In conclusion, the marked differences between the mammalian and piscine immune systems and the relative lack of specific reagents may serve to explain why fish vaccine testing still mainly relies on in vivo methods, and why the development of alternatives based on serology or antigen quantification test is slow.

\subsection{Pain, illness and suffering in fish}

If it is accepted that fish have an awareness of pain, it is important to understand how experimental procedures may affect them, so that any suffering can be prevented or minimized [21]. This section defines pain, for the purposes of this report, and discusses the evidence for pain perception and suffering in fish as it is believed to occur during vaccine research and quality control. Pain can be defined as a negative sensory and psychological experience. This splits pain into two components, where there is (i) tissue damage giving rise to the sensation of pain and (ii) a negative subjective experience, or suffering [22]. Judgments about the capacity of non-human animals to experience pain must be made for each species using behavioral, physiological and neurological indicators as a basis. For example, some pain 
research involves recording behavioral and physiological responses to a potentially painful event. If normal behavior is suspended and negative or abnormal changes in behavior and physiology occur, then it can be inferred that the animal is experiencing a negative affective state alongside the sensory component.

There are a number of other techniques that can be used to deduce whether animals perceive pain. Examples are investigating whether the species has the neural machinery (nociceptors) to detect noxious stimuli that give rise to the pain sensation, whether analgesics reduce behavioral and physiological responses to a potentially painful event, and whether animals can learn to avoid damaging stimuli.

\subsubsection{Sentience, consciousness and cognition of pain}

A number of studies have been conducted using the above techniques to assess the potential for nociception and pain in fish. It has been shown that fish do have nociceptors that detect painful stimuli [23], with physiological properties that are very similar to those found in mammals [24-28]. In several fish species activity patterns differ when the animals are subjected to non-noxious stimuli as opposed to painful stimuli $[21,29,30]$. The use of morphine in trout given a potentially painful stimulus decreased anomalous behaviors and prevented the suspension of normal behavior [31]. Fish are capable of learning to avoid noxious stimuli and this learning does not occur when morphine is administered, suggesting that pain is reduced [32]. Finally, noxiously stimulated trout do not show an appropriate response to a fear test and this suggests that pain dominates their attention and is therefore an important and unpleasant state to the fish $[33,34]$.

While there is evidence for nociception in fish, an animal has to be sentient or conscious in order to experience pain. Sentience is the ability to perceive external stimuli and research has shown that fish can sense potentially painful stimuli and that their behavior is negatively affected by such stimuli, therefore, it could be argued they are aware of/can experience pain. "Higher order" forms of consciousness include self-awareness, meta-cognition (having thoughts about thoughts and creating internal representations) and finally episodic memory where animals can think about the past and future. Evidence for these higher order forms have been demonstrated in birds, rats, dolphins and primates [35-39] but have never been explored in fish and so it cannot be stated for certain that they do or do not exist.

Therefore, the participants in the workshop do not propose that fish have a similar experience of pain to humans, but conclude that fish do experience noxious stimuli in a way that has a detrimental effect on their behavior, physiology and well-being. It is therefore important that fish are treated in a humane manner, including during experimentation. In the case of vaccine research and quality control, this especially means minimizing and alleviating any pain caused by procedures that might cause tissue damage or by illness that may develop.

Inferring from human experience, one can argue that most generalized infections are not painful per se. Suffering is mainly associated with a general feeling of weakness and poor wellbeing, abnormality in physiological functions, negative feelings and anxiety, including the fear of permanent disability or death. Thus it appears that suffering in many illnesses is closely associated with the cognitive and mental capacities of the sufferer. For the purpose of this report, however, and irrespective of potentially different views upon the mental capabilities of fish, it was agreed that inflicting a generalized infection upon experimental fish should be regarded as an invasive procedure with the potential to cause suffering. Experimental infection may cause weakness, poor wellbeing, clinical illness, tissue damage and eventually death. It is therefore important to be able to recognize signs of illness in fish so that humane endpoints can be implemented if possible (see 4.4 Welfare assessment). 


\subsection{Animal tests stipulated for the production and quality control of fish vaccines}

The regulatory authorities in Europe and North America stipulate animal tests to achieve approval for marketing a vaccine and for batch (serial) release of the authorized vaccine.

A detailed description of the regulatory framework, the various requirements and animal tests are given in Appendix A.

The various tests are briefly summarized and the minimum numbers of animals required are listed in Table 1.

\subsubsection{Efficacy testing (prior to product marketing authorization)}

Fish of the target species are vaccinated in the conditions to be recommended for use. They are challenged together with the unvaccinated control fish via a specific route and at a fixed interval after vaccination. The fish are observed until at least a given percentage of specific mortality is reached in the control group. The relative percent survival (RPS) values are calculated and have to meet the specified regulatory requirements.

\subsubsection{Safety testing (prior to product marketing authorization)}

The safety of fish vaccines for all recommended uses has to be demonstrated under laboratory and field conditions. Fish are vaccinated and, for a given period, observed for local and systemic reactions.

\subsubsection{Batch (serial) potency testing}

Batch/serial potency can be either by a vaccination-challenge test (as described above) or an antibody induction test, for which fish are vaccinated and the level of antibodies induced is measured with immunochemical methods. Under certain conditions, in vitro methods (e.g. antigen quantification) can be used for serial potency testing in the US.

\subsubsection{Batch (serial) safety testing}

According to the European Pharmacopoeia (Ph.Eur.) monographs and European guidelines, the batch safety test is carried out with a double dose of the vaccine administered to fish by the recommended route and observed for a given period. No fish should show notable signs of disease or die from causes attributable to the vaccine. As stated in the Ph.Eur. general monograph on veterinary vaccines, the routine application of the batch safety test can be waived for established vaccines in agreement with the competent authority provided that consistency of production has been demonstrated for at least 10 consecutive batches.

Batch (serial release) safety testing of fish vaccines in the US may be conducted by observation of fish after vaccination during vaccination-challenge or vaccination-serology studies. Separate batch safety studies in fish are required when potency is demonstrated by in vitro methods or in a non-target host (i.e. in a species other than that for which it is recommended).

\section{Potential for replacement}

The highest number of experimental fish is currently being used during the quality control of fish vaccines prior to batch release. Replacing in vivo with in vitro methods for quality control of commercial batches of vaccines therefore has the potential to significantly reduce the numbers of fish used. 
Following the consistency approach ([40], see also below), the focus of development and licensing, including the documentation of safety, may be shifted toward a more comprehensive documentation of both, acute and chronic systemic and local reactions. The demonstration of consistency with regard to product safety and efficacy, along with prioritized development and validation of alternative potency test methods, would allow for the total replacement of animal testing for batch release. If an alternative potency test method can be validated before full licensing, there would even be an opportunity for the elimination of animal testing in the demonstration of product stability.

Table 1. Minimum numbers of animals stipulated for the tests required in Europe and North America.

\begin{tabular}{|c|c|c|c|c|}
\hline & Ph.Eur. & $\begin{array}{c}\text { EU } \\
\text { guidelines }\end{array}$ & Canada & USA \\
\hline \multicolumn{5}{|c|}{$\begin{array}{l}\text { Prior to product approval } \\
\text { for marketing }\end{array}$} \\
\hline Efficacy & 200 & Not given & 100 fish per challenge organism & $\begin{array}{l}\text { Usually at least } 3 \\
\text { replicates of } 50,150 \\
\text { total }\end{array}$ \\
\hline $\begin{array}{l}\text { Safety } \\
\text { Laboratory }\end{array}$ & 50 & Not given & $\begin{array}{l}0 \text { (carried out in combination with, } \\
\text { i.e. pre-challenge part of the } \\
\text { efficacy) }\end{array}$ & $\begin{array}{l}0 \text { (carried out in } \\
\text { combination with } \\
\text { efficacy) }\end{array}$ \\
\hline Field & $\begin{array}{l}180 \text { or a sufficient number } \\
\text { of fish distributed in not } \\
\text { fewer than } 2 \text { sets of } \\
\text { premise }\end{array}$ & & $\begin{array}{l}\text { no minimum, require safety testing } \\
\text { of multiple serials at multiple } \\
\text { geographical locations) }\end{array}$ & 1000 \\
\hline \multicolumn{5}{|c|}{ Batch/serial testing } \\
\hline $\begin{array}{l}\text { Potency } \\
\text { Challenge } \\
\text { Serotology }\end{array}$ & $\begin{array}{l}60 \\
35\end{array}$ & Not given & $\begin{array}{l}50 \text { fish per challenge organism. In } \\
\text { vitro potency acceptable if properly } \\
\text { validated }\end{array}$ & $\begin{array}{l}\text { In vitro potency } \\
\text { allowed for batch } \\
\text { release; challenge or } \\
\text { serology allowed but } \\
\text { not required }\end{array}$ \\
\hline Safety & 10 & 30 or 50 & $\begin{array}{l}0 \text { (carried out in combination with } \\
\text { efficacy, i.e, pre-challenge part } \\
\text { of the efficacy) }\end{array}$ & 25 \\
\hline
\end{tabular}

\subsection{Batch potency testing alternatives based on antigen quantification}

New concepts of quality control can offer alternatives to current vaccination-challenge models.

The "consistency approach" is based on the principle that the extent of vaccine batch release testing should reflect the level of consistency achieved during the production process. It implies the use of a set of in vitro measurable parameters (e.g. antigen content and integrity) to constitute a product profile that can replace or reduce current release tests. This product profile ensures that each batch is in diverse aspects similar to a manufacturer-specific vaccine of well-documented efficacy and safety. Monitoring of physiochemical parameters (e.g.pH value, viscosity, amount of inactivating and/or preservative agents) will serve to verify consistency of the production process [40]. Gains need to be made to consider batch potency by antigen quantification on the key attributes for an acceptable test. There may be an improved validation potential, increased reproducibility, differentiation of potent from sub-potent batches, and utility to employ the test throughout the shelf-life of the product in the same test system over models involving animals. 
A good alternative to a vaccination-challenge test would be to establish methods (e.g. ELISA) for relative potency testing by antigen quantification. Such assays should be developed to measure an antigen or antigens that are immunologically functional and can be quantified in the final product:

\subsubsection{Technical considerations}

a. The assay could be used both for in-process control and formulation, and also as a potency test throughout the shelf-life of the product. Such an assay requires the use of a reference vaccine which has been proven to be efficacious in the target animals. The ELISA would be standardized to utilize specific antibodies to capture the antigen and quantify the concentration [41]. The estimation of batch potency should be based on a "relative potency approach" and has to be in line with the requirements outlined in Ph.Eur., Chapter 5.3. "Statistical analysis of results of biological assays and tests" [42].

b. The potential for modulation of other antigens in a multivalent formulation might not be captured in every type of antigen quantification assay, but can be controlled with additional antigen-based tests and specifications on the concentrations of all other antigens in a defined product. Tighter specifications for quantification of bacteria or virus particles in vaccines by total particle counts (cells or antigenic units) for example will make this more feasible.

c. The antigen quantification test should be validated according to $\mathrm{VICH}$ Guideline 2 [43]. During validation it should be demonstrated that the chosen potency test is capable to distinguish between standard and sub-potent batches on the basis of antigen content.

\subsubsection{Physico-chemical considerations}

d. Antigen recovery experiments will show, whether elution is required to quantify the antigen in adjuvanted products. e. In addition, the batch control testing would also require an assay to estimate the consistency of the final product formulation. Through the validation studies, potential effects of antigen-adjuvant formulation such as modulation of the immune response, vaccine deposition and slow release, or antigen presentation should be addressed.

f. The inactivating and/or preservative agents used in manufacture must be considered during validation of an ELISA assay for antigen quantification. The agents may be present in the final formulation of the vaccine and the effect and antigen retention capacity of those agents may change over time.

\subsubsection{Immunochemical considerations}

g. The ELISA assay should employ a reference batch of Good Manufacturing Practice vaccine produced to the antigen specification as described in the dossier for licensing. Samples of the reference vaccine must be kept without degradation and a defined period for use of the reference vaccine should be determined. Its stability should be monitored in regular intervals and appropriate warning and action limits should be set. A replacing procedure should be established. The reference vaccine would be made from an antigen where the dose and function were determined. An immunogenicity study of the reference vaccine should be established by validation of potency in an in vivo vaccination-challenge model as required in Directive 2001/82 as amended [44].

h. While the quantification of protective antigens is optimal, any antigen that is capable of creating an immunological response may be relevant. During development phases, several methods could be employed to define a protective or immunorelevant reference antigen for use in ELISA tests. The 
antigen selection could be optimized by harvesting immunoglobulin from convalescent or immunized target animals in an in vivo model. Using an immune complexing method with the harvested antibody, one could isolate and enrich immunorelevant proteins from a cell lysate of the vaccine preparation. Further isolation of key antigens could be done using $2 \mathrm{D}$ electrophoresis gels for identification of single proteins that might be further characterized by mass spectrometry. Other serological evaluations such as immunoblotting could also be used to capture immunorelevant antigens to select for a monitoring antigen in an alternative assay using monoclonal or polyclonal antisera.

i. Where antigens in the vaccine are derived from crude cell lysates or whole cell preparations, the use of polyclonal sera could also be used in anantigen quantification assay to measure the relative quantities of all immunorelevant antigens without specific characterization. The rationale of a batch potency test is to demonstrate consistency of antigenic properties and an immunological function achieved by an immunological assay. For each test, the specific criteria that the products have to meet for antigen quantification would need to be determined. These specifications may need to be determined on a per product basis because no internationally standardized reagents are available now or in the foreseeable future.

Recommendations:

- Regulators should encourage the development, validation and use of antigen quantification methods for batch potency testing and incorporate them into the respective guidelines and monographs.

- The potential of the consistency approach and its application to the batch testing of fish vaccines should be evaluated.

\subsection{Waiving the use of primary cell lines for extraneous agent testing of master seeds}

The participants in the workshop believe that for the development of a fish vaccine, the use of fish to isolate de novo primary cells for extraneous agent testing of master seeds should not be required. The use of animals to create this primary cell culture(with the need to recover tissues from multiple fishes, multiple times in order to gain the number of cells required at a low passage number) does not make scientific sense. The interpretation of the relevant guidance documents [45] by medicinal authorities is that there needs to be testing of the master cells and seeds on a primary cell line from the species of origin. A primary cell line is one which is described as only one and preferably no more passages from the primary isolation. An argument for the use of primary cell lines is that unknown extraneous viruses could be better detected because primary cell lines contain a more diverse range of cell types, thus (potentially) increasing the chance that at least one of them may be permissive. However, even primary cell lines are normally of one cell type (such as epithelioid cells) and will thus not provide additional probability of extraneous agent detection. For fish, especially for salmonids, there are several continuous cell lines from the species of origin that could be used to test for extraneous agents. Any cell culture used for extraneous agent testing should ideally itself be shown to be free of extraneous agents, which is not possible when using primary cell cultures.

Recommendation:

- Continuous cell lines should be allowed for extraneous agent testing of master seeds. 


\section{Potential for reduction}

The greatest potential to reduce the number of fish used for fish vaccine testing lies in the omission of certain tests that are of doubtful value to assure the quality, efficacy or safety of the products. These are discussed below.

Apart from this, high priority should be given to reducing the numbers of fish subjected to the most severe procedures or to procedures inflicting clinical illness on the experimental animals. Most likely these are the control fish in vaccination-challenge tests, since they are unprotected and are -- by the very nature of the test -- expected to develop disease and, if it is not possible to implement humane endpoints, they may eventually die as a result.

Recommendation:

- Priority should be given to reducing the numbers of fish subjected to procedures that may cause severe outcomes.

\subsection{Re-testing for batch release in Europe only when scientifically justified}

Current European legislation requires that for veterinary medicinal products produced outside the European Economic Area, each batch imported to a Member State must undergo a full qualitative analysis, a quantitative analysis of at least all the active substances, and all other tests or controls necessary to ensure the quality in accordance with the requirements of the marketing authorization [44]. There is no scientific rationale for this when it comes to most fish vaccines imported into Europe, bearing in mind that target species and breeds (Atlantic salmon, rainbow trout) as well as the environmental conditions may be identical or essentially similar in Europe as in the country of origin. Acceptance of batch safety and potency data generated in the country of origin would therefore have a significant potential for reducing the overall numbers of fish used for fish vaccine testing.

Recommendation:

- Vaccines produced outside the European Economic Area should be re-tested for batch release in Europe only when scientifically justified. Alternatively, the manufacturers from outside Europe should organize that batches intended for the European market are tested only once at a certified testing site within the European Economic Area.

\subsection{Documentation of product safety for licensing}

It is necessary to fully assess and document the safety of a vaccine during product development. This requires extensive investigations on toxicological, systemic and local reactions, behavioral and physiological parameters, feeding, and growth. At present, double-dose safety trials are a legal requirement for the licensing of an inactivated fish vaccine. However, the benefits of double-dose trials vs. single-dose experiments to yield relevant safety data have been questioned. If samples and observations from single-dose experiments are shown to yield satisfactory safety documentation, relevant dual purpose data (safety and efficacy) could be derived from the same single-dose experiments.

\section{Recommendation:}

- The Ph.Eur. monograph requirements for documentation of target animal vaccine safety should be re-assessed for fish vaccines, giving consideration to the VICH Guideline 44, that was adopted during 2008 [46]. 


\subsection{Safety testing for batch release}

The current European requirements stipulate a double-dose target animal batch safety test (TABST) in at least 10 fish (according to Ph.Eur. monographs) or at least 30 fish (according to EU guidelines). The reasoning behind this discrepancy remains unclear. The EU guideline on specific requirements for fish vaccines is currently under revision and this issue should be addressed during the revision.

Since 2005, the Ph.Eur. general monograph on veterinary vaccines [47] allows waiving of the batch safety test for established vaccines in agreement with the competent authority provided that consistency of production has been demonstrated for at least 10 consecutive batches. The CVMP has further elaborated this issue in its position paper on data requirements to remove the TABST [48]. Despite this possibility of reduction, manufacturers are reluctant to apply it as outlined in a review document on animal welfare progress in Ph.Eur. monographs [49]. Reasons for this are the lack of harmonization between European competent authorities and with regions outside of Europe.

Recommendations:

- European regulators should harmonies their criteria for waiving the batch safety test.

- The VICH should work toward harmonization of the requirements for the batch safety test for veterinary vaccines including fish vaccines. ${ }^{1}$

In addition, the workshop participants suggested a different approach to batch safety testing, since the current safety test in at least ten animals is not determinative for the safety of adjuvanted fish vaccines and thus has questionable relevance for product quality. If single-dose testing would be allowed, data and observations derived from the vaccination of animals as part of the current in vivo potency test could be used for documentation of batch safety. For this purpose, specific parameters for recording safety relevant outcomes of single-dose immunization would need to be developed and validated. This should take into account the requirements outlined in the CVMP position paper "On data requirements for removing the target animal batch safety test for immunological medicinal products in the EU" [48].

In cases where a validated antigen quantification method is employed for potency testing, a separate single-dose safety test would have to be conducted for a certain number of consecutive batches to demonstrate consistency of production.

\section{Recommendation:}

- The approach to batch safety testing should be revised, i.e. if single-dose safety testing would be possible, relevant data on safety could be obtained through observations of the single-dose immunized fish used for batch potency testing. ${ }^{2}$

\subsection{Waiving animal use to document the seronegative status of test fish}

There is currently a requirement to document that fish used for safety testing of certain vaccines are seronegative. However, there are no reports describing the phenomenon of anaphylaxis in fish, nor of other biological effects on adverse vaccine reactions induced by the presence of antibodies to vaccine antigens [50]. In vaccines not based on toxoids, antibodies are considered unlikely to interfere with tolerance. The relevance of the requirement to prove that fish are seronegative may therefore be questioned. The omission of this requirement would, however have only a minor effect on reduction and

\footnotetext{
${ }^{1}$ Ongoing activities to remove the target animal safety test from. Ph.Eur. monographs (status 2010).

${ }^{2}$ Ongoing activities at $\mathrm{VICH}$ level on criteria for waiving the target animal safety test (status 2010).
} 
refinement, because testing is normally performed on entire recruitment populations (from which fish are drawn for multiple batch tests), and sampling is performed after sedation and stunning.

The requirement for documenting the seronegative status of fish to be used for potency testing of Vibrio vaccines (or other vaccines against strictly marine pathogens) should be maintained only in cases where the test animal population has been exposed to seawater. Test animals of strictly freshwater origin are unlikely to have been exposed to these pathogens, and documentation to substantiate non-exposure to saltwater systems should be deemed sufficient to waive this requirement.

Recommendations:

- Documentation of the seronegative status of fish for safety testing should be eliminated from all fish vaccine monographs as the serological status is considered irrelevant to safety.

- Documentation of the seronegative status of fish for potency testing of strictly marine pathogens (e.g. Vibrio spp.) should be waived when it can be demonstrated that test fish had not been exposed to seawater.

\subsection{Optimize vaccinated/control fish ratio}

The monograph requirements stating the minimum number of fish to be involved in efficacy documentation and batch potency tests should be re-assessed, aiming to minimize the number of unprotected control fish without compromising the statistical power of the tests. This work could build upon previous work addressing both statistical andbiological aspects of fish vaccination trials [50-52] and should also include scrutiny of pass criteria such as the estimates of relative protection (RPS). A vast amount of recordings could potentially be made available in anonymous form by fish vaccine manufacturers, creating a unique database for retrospective analysis, and allowing the use of practical experience as the basis for statistical assessment and optimization of test design.

Recommendation:

- The potential to reduce the number of control fish used in vaccination-challenge tests for efficacy and batch potency testing should be evaluated, since most of these unprotected fish are subjected to development of disease and associated distress.

\subsection{Use of genetically homogenous experimental fish strains}

In several experimental animal species, the use of genetically homogenous animals (inbred strains) is being employed to reduce within-group variability and thus to minimize the number of individuals necessary for biomedical experiments [53]. While outbred populations of fish species such as salmon, rainbow trout and carp are known to be immunogenetically diverse [54-56], inbred or isogenic strains of major aquaculture species are not currently available. Thus, the use of inbred strains is unlikely to contribute much to reduction in the near future. However, the workshop participants strongly encourage innovative academic research to assess the principal potential of genetic homogenous fish for reducing fish numbers in biomedical testing in the long-term perspective.

Recommendation:

- Research should be conducted into (i) the potential to produce inbred strains of fish, and (ii) the contribution that these strains could make to reducing fish use and improving scientific validity in biomedical research, including vaccine development and production. 


\section{Potential for refinement}

Refinement has been defined as "any approach which avoids or minimizes the actual or potential pain, distress and other adverse effects experienced at any time during the life of the animals involved, and which enhances their wellbeing" [57]. The concept of refinement thus means considering the animal's whole lifetime experience, not just reducing suffering during scientific procedures.

In the case of fish used in vaccine studies, important causes of suffering or poor welfare are the effects of disease challenge, the side effects of vaccines (both predictable and unpredictable) and pain arising from scientific or husbandry procedures. For example, besides undergoing an invasive procedure, fish subjected to inoculation challenge will likely (even if fully or partially protected) be at least temporarily affected by exo- or endotoxins of the inoculum, and of bacteriaemia/viraemia, with corresponding physiological changes. At the other end of the scale, inoculation procedures performed under anesthesia, for example single-dose or even double-dose immunization for safety assessment, are believed to cause minimal pain or suffering in the experimental fish.

The participants in the workshop assumed that fish may experience pain as a result of tissue damage, but the potential for fish to suffer due to the effects of toxins or disease has not been objectively evaluated at the time of writing. Therefore, it was agreed that fish are given the benefit of the doubt and that inflicting illness should be considered undesirable from an animal welfare viewpoint.

\subsection{Batch potency testing alternatives based on serological methods}

Serological methods (specific antibody response measurements) are a potential way of reducing the number of fish routinely being subjected to experimental challenge procedures. The option is already permitted in Ph.Eur. fish vaccine monographs. However, little progress has been made in the development and validation of such alternative batch potency tests. A project group has recently showed promising results in developing a batch potency test for oil adjuvanted furunculosis vaccines in Atlantic salmon by antibody ELISA (AB Romstad, pers. comm. during this workshop). A time point for blood sampling has been determined and the potential of the test to reveal sub-potent batches has been evaluated. Further work is needed to investigate whether the method can be used for the testing of multivalent vaccines, to evaluate intra- and interlaboratory variability, and to establish whether the measured antibodies in themselves are protective (by passive transfer).

Especially for multivalent vaccines, up to six groups of fish used in parallel challenge procedures (one for each vaccine antigen) could be saved by using the same blood samples from one immunized group to confirm batch potency for all antigens. However, given the paucity of academic research on such tests and the economic limitations of the fish vaccine market, the costs of developing and validating such alternative batch test methodologies are prohibitive on a product-by-product basis. This work should be encouraged and sufficient funding should be mobilized to develop and validate methods for such batch tests and to provide the necessary reagents. This would stimulate vaccine manufacturers to adapt such protocols by inclusion of product-specific key reagents (e.g. antigens for coating ELISA plates, monoclonal antibodies, etc.).

Obviously, the use of serological methods would also contribute to the refinement of fish vaccine trials, as the most invasive among the current trial procedures (experimental challenge) is no longer needed.

Recommendation:

- Efforts should be undertaken to develop and validate serological methods for batch potency testing. 


\subsection{Procedures}

Procedures that may cause discomfort, pain or distress in fish vaccine studies include handling, identification, administration of substances in the case of challenges by injection, and killing. Guidance on refining some common procedures in fish can be found in the Canadian Council on Animal Care "Guidelines on: the care and use of fish for research, teaching and testing" [58].

\subsubsection{Analgesia/anesthetics/sedation}

The use of analgesics, anesthetics and sedatives to reduce suffering during potentially painful or stressful procedures in fish should always be given full consideration. Decisions on whether these agents will be used should be based on judgment about the invasiveness of the procedure and the benefits of anesthesia or analgesia against any potential harm associated with the analgesic, anesthetic or sedative itself. For example, there may be trauma associated with delivery, side effects or recovery time. There is also evidence that some anesthetic agents can be aversive and stressful to fish [59]. A range of potential agents should be considered and expert advice sought in relation to their relative aversiveness, refinements in delivery and whether there is evidence for or against any genuine impact on scientific outcomes. Analgesia, anesthesia or sedation should be used whenever their benefits are considered to outweigh the potential harms to the fish and their use will not compromise the science (For further guidance see [58]).

Examples of procedures where analgesics, anesthetics or sedatives are often used in fish vaccine testing are handling, identification, transport and euthanasia. The effect of analgesics on the immune response has not been evaluated in fish, but it may be possible in many cases to allow the agent to wash out before beginning scientific procedures. In addition, unrelieved pain will also have an effect on the immune response and thus on animal physiology.

\section{Recommendations:}

- Analgesics, anesthetics or sedatives should be used during any potentially painful procedure or stressful event, whenever the benefits are predicted to outweigh any potential harm due to administration trauma or side effects and the science will not be compromised.

- More research is needed into the effects of sedatives and analgesics on the immune responses of fish, to alleviate concerns about interference with study objectives.

\subsubsection{Handling}

Handling is likely to be highly stressful for fish, especially if netting and holding in air is involved. It may be possible to avoid exposure to air, when transporting fish between tanks for example, by moving them in containers in water-filled containers.

Recommendation:

- Fish should preferably be kept in water whenever being moved

- Exposing fish to air during handling and transport should be avoided wherever possible.

\subsubsection{Marking methods and identification of fish}

Marking individual fish for identification purposes will inevitably cause a degree of distress and discomfort, because it involves capture and handling as well as applying the chosen technique. Table 2 lists currently available techniques for identifying fish, with brief information to enable the least invasive technique to be 
chosen that is compatible with the scientific aims, any requirement to reduce numbers and the equipment and staff skills that are available (see also [58]).

Note that in some instances there can be a dilemma between refinement and reduction, for example using Passive Integrated Transponder (PIT) tags for identification requires invasive procedures but can enable the number of experimental animals to be reduced. In general, the aim should be to minimize suffering and maximize welfare of individual animals even if more animals may be used.

Recommendations:

- Identification is a potentially painful and distressing procedure and therefore current knowledge about available techniques should be researched and the least invasive method chosen that is appropriate, taking into account any potential to reduce animal numbers.

- It should be ensured that everyone who will be marking fish is appropriately competent and empathetic, has adequately functioning equipment and a realistic workload.

\subsubsection{Administration of substances/challenge organisms}

Substances or challenge organisms may be administered to fish using a number of routes in vaccine test studies such as injection, orally and by immersion. Administration by injection involves handling stress, the discomfort of the injection, possible pain due to tissue distension or the physico-chemical nature of the substance or challenge organism, and potential adverse after-effects of the substance or organism [60]. Less invasive administration techniques are to be preferred wherever possible; for example, infecting a small number of fish via waterborne exposure (ideally cohabitation) and allowing them to challenge other test fish is a commonly used refinement in vaccine studies. Fewer animals might be affected by toxic components of the challenge organism and/or temporary or permanent bacteriaemia/viraemia when administration is done via the water, compared to administration via inoculation. General guidance on refining the administration of substances was published by Morton et al. [61] and the CCAC [58].

Recommendations:

- The least invasive or least stressful technique for administering substances or challenge organisms to fish that is compatible with the aims of the experiment should be used.

- Infection by immersion, bath or cohabitation should be considered as an alternative to invasive administration techniques.

- Ways to further refine the chosen method should be researched, for example by reducing needle gauge, improving dosing technique or selecting a less irritant carrier.

\subsubsection{Euthanasia}

New EU legislation ${ }^{3}$ states tha tmost fish should be killed either by concussion of the brain by striking of the cranium, or by an overdose of anesthetic using an appropriate route and anesthetic agent for the size and species. Electrical stunning is permitted provided that specialized equipment is used. When killed by anesthetic immersion, fish should be left in the solution for at least $5 \mathrm{~min}$ following the cessation of opercular movement and/or vestibulo-ocular reflex. In both cases, death should be confirmed, for

${ }^{3}$ At the time of the workshop the revision of Directive 86/609/EEC on the protection of laboratory animals was ongoing (http://ec.europa.eu/environment/chemicals/lab_animals/home_en.htm). The new Directive 2010/63/EU on the protection of laboratory animals was published on 22 September 2010. 
example, by physical destruction of the brain or exsanguination [62]. The relative humaneness of physical and chemical methods, and the aversiveness of different anesthetic agents, has not been evaluated for fish [63]. However, some techniques used for killing fish in non-laboratory settings are clearly inhumane and should never be used in vaccine research, such as suffocation in air, chilling on ice or tearing gills (See also $[58,63]$ and the Norwegian School of Veterinary Science website; http://oslovet.veths.no/[click on "fish" and "euthanasia"]).

Table 2. Techniques for marking individual fish.

\begin{tabular}{|c|c|c|c|}
\hline Technique & Description & Welfare advantages & Welfare disadvantages \\
\hline $\begin{array}{l}\text { Dye or elastomer } \\
\text { injection }\end{array}$ & $\begin{array}{l}\text { Small amount of dye, colored } \\
\text { polymer or colored latex injected } \\
\text { into the skin }\end{array}$ & Minimally invasive & $\begin{array}{l}\text { Handling stress, injections may } \\
\text { be painful }\end{array}$ \\
\hline $\begin{array}{l}\text { Passive Integrated } \\
\text { Transponder } \\
\text { (PIT) tags }\end{array}$ & $\begin{array}{l}\text { Small "chip" with unique } \\
\text { identifying numbers inserted into } \\
\text { the body cavity or muscle using a } \\
\text { trochar or needle }\end{array}$ & $\begin{array}{l}\text { Can lead to reduction -- allows } \\
\text { more sophisticated experimental } \\
\text { design; potential to use } \\
\text { automatic readers so no } \\
\text { subsequent handling required }\end{array}$ & $\begin{array}{l}\text { Invasive technique into abdomen } \\
\text { or deep into tissues }\end{array}$ \\
\hline $\begin{array}{l}\text { External tags } \\
\text { (Floy-tags etc.) }\end{array}$ & $\begin{array}{l}\text { Colored or numbered tag inserted } \\
\text { into musculature, often just below } \\
\text { dorsal fin }\end{array}$ & $\begin{array}{l}\text { Can lead to reduction -- allows } \\
\text { more sophisticated experimental } \\
\text { design }\end{array}$ & $\begin{array}{l}\text { Handling stress, discomfort, } \\
\text { Permanent tissue penetration } \\
\text { may cause ulceration and } \\
\text { infection }\end{array}$ \\
\hline "Tattooing" & $\begin{array}{l}\text { Pigment is injected into skin cells } \\
\text { using high-pressure air device } \\
\left.\text { (Panjet }{ }^{\mathrm{TM}}\right)\end{array}$ & $\begin{array}{l}\text { Long-lasting, rarely causing } \\
\text { damage to dermal tissues }\end{array}$ & $\begin{array}{l}\text { Can deteriorate over time } \\
\text { triggering need to be repeated }\end{array}$ \\
\hline Freeze branding & $\begin{array}{l}\text { Metal brand cooled to below } 0^{\circ} \mathrm{C} \\
\text { held against the skin for } 3-5 \mathrm{~s}\end{array}$ & $\begin{array}{l}\text { Long-lasting, no need for further } \\
\text { handling or reapplication }\end{array}$ & $\begin{array}{l}\text { Causes tissue necrosis Not } \\
\text { recommended in cold water as } \\
\text { healing time increased and } \\
\text { secondary infections with } \\
\text { ulceration can occur }\end{array}$ \\
\hline Fin clipping & Part of a fin is removed & $\begin{array}{l}\text { Long-lasting, no need for further } \\
\text { handling or reapplication }\end{array}$ & $\begin{array}{l}\text { Nociceptors are present on fins } \\
\text { so could be painful; Healing } \\
\text { may result in poor discrimination } \\
\text { not recommended in cold water }\end{array}$ \\
\hline Fin removal & An entire fin is removed & $\begin{array}{l}\text { Long-lasting, no need for further } \\
\text { handling or reapplication }\end{array}$ & $\begin{array}{l}\text { Invasive } \\
\text { handling } \\
\text { required }\end{array}$ and $\quad \begin{array}{l}\text { technique } \\
\text { anesthesia }\end{array}$ \\
\hline Adipose fin clip & $\begin{array}{l}\text { Removal of adipose fin } \\
\text { appendage }\end{array}$ & $\begin{array}{l}\text { Non-invasive to minimally } \\
\text { technique }^{\mathrm{a}}\end{array}$ & $\begin{array}{l}\text { Handling and anesthesia } \\
\text { required, allows only } 2 \text { groups }\end{array}$ \\
\hline $\begin{array}{l}\text { Fluorescent dyes of } \\
\text { scales }\end{array}$ & $\begin{array}{l}\text { Immersion in fluorescent } \\
\text { substance }\end{array}$ & Non-invasive technique & $\begin{array}{l}\text { Some handling required, few } \\
\text { groups only }\end{array}$ \\
\hline $\begin{array}{l}\text { Otolith marking using } \\
\text { rare } \\
\text { earth elements }\end{array}$ & $\begin{array}{l}\text { Immersion or oral administration } \\
\text { of marker } \\
\text { identification by biochemical } \\
\text { analysis }\end{array}$ & Non-invasive technique & $\begin{array}{l}\text { Fish must be euthanized before } \\
\text { reading }\end{array}$ \\
\hline
\end{tabular}


Recommendations:

- It should be ensured that fish are only killed by concussion, anesthetic overdose or electrical stunning as described above, by competent staff with an appropriate workload.

- More research is needed into the humaneness of different techniques for killing fish of various sizes.

\subsection{Environment}

There are two key components in providing fish with an appropriate environment that will facilitate good health and welfare and minimize stress; (i) ensuring good water quality and appropriate water temperature and (ii) providing a stimulating environment that will encourage a range of natural behaviors.

\subsubsection{Water quality and water temperature}

Good water quality is absolutely essential for ensuring fish health and welfare. General guidance on water quality, including temperature and levels of oxygen, nitrogen compounds, carbon dioxide, $\mathrm{pH}$ and salinity, can be found within EU legislation [62,64] and the CCAC guidelines [58]. Precise requirements can vary between species and within the life cycle of individuals, so it is vital to research and provide the optimum water quality for each species.

In salmonids, there is evidence that the side-effect potential of oil adjuvanted bacterin formulations may unfold more rapidly and vigorously at higher temperatures, i.e. at $17^{\circ} \mathrm{C}$ than at $10-12^{\circ} \mathrm{C}$ [65]. However, by elevating the water temperature, injection-site inflammatory lesions will be more severe. Research should be carried out to investigate the potential benefits of changing the temperature recommendations, and how this may lead to reduction in the number of fish used without compromising the power of these trials to reveal unfavorable product characteristics, or batches falling outside the acceptable safety limits. Such research should also address the ethical dilemma of reducing fish numbers vs. the potentially increased severity of reactions in each test animal used.

\subsubsection{Enrichment}

Environmental enrichment has been shown to encourage a range of natural behaviors and reduce stress in a range of species, which can have both animal welfare and scientific benefits. Enrichment commonly provided for fish used in research and testing includes variable water flow, tank shading, variety of foods, plants (real or artificial), species-appropriate substrate, refuges (e.g. terracotta pots, netting for fry, artificial nests for breeding) and rocks $[66,67]$. However, there may be complicating factors in the field of veterinary vaccine studies due to the need for stringent hygiene standards in some tests.

Despite constraints in some studies, it should still be possible to design an environment that affords a degree of security and stimulation. For example, security can be provided for by adding refuges or by covering the tank with a solid lid, including transparent areas or portholes to allow the fish to be observed and monitored without lifting the lid. Fitting a lid can also help to reduce animal numbers, as fish will be unable to jump out of the tank. Tinted tanks, artificial water lily pads and plastic plants are other options. It is also important to research the natural biology and behavior of the test species when deciding on appropriate enrichment; for example pelagic (schooling) and riverine (territorial) fish may have different needs.

For further guidance on fish husbandry see: [58,68,69 and http://oslovet.veths.no/(click on "fish" and "guidelines")]. 
Recommendations:

- It should be ensured that water quality and temperature are always within appropriate physiological limits.

- Species-appropriate environmental enrichment should be provided wherever possible.

- Fish should be housed in appropriately sized and composed groups to facilitate species-specific social behavior wherever protocols permit.

- Restrictions on enrichment should be questioned and creative alternative approaches to provide an appropriate environment should always be sought.

\subsection{Welfare assessment}

An essential part of refinement is better recognition and assessment of the causes and effects of animal suffering. This helps to improve the prevention and alleviation of any pain, suffering or distress due to procedures as well as other effects such as anxiety. Welfare assessment is heavily dependent on interpreting animal behavior, but this can be especially difficult in the case of fish because their behavior is very different from that of mammals. In addition, it can be difficult to monitor individuals if very large numbers are housed together.

An effective welfare assessment system is essential for helping to identify behaviors that are a cause for concern and for detecting signs of incipient suffering, thereby enabling humane endpoints to be implemented (see below). The ideal system will include as many objective indicators as possible (e.g. food consumption), consist of a small number of criteria that are easy to identify and avoid the observer having to make a veterinary diagnosis as part of the assessment.

\subsubsection{Signs of illness}

In response to generalized bacterial or viral infections, fish often show an enhanced respiration rate (i.e. more frequent opercular beats), reluctance to feed, abnormal (passive) behaviors and a reduction in activity as the first signs of illness [26,30]. Other examples of clinical signs that are commonly used in practice include skin color (especially darkened skin), red gills, decreased opercular beat rate, eye color, physical injury, physical abnormalities and changes in oxygen demand [67]. Physiological indicators are of limited use, because they are very difficult to measure without disturbing the fish, only a few have been documented, and many of them depend on water temperature. Behavioral signs that could indicate welfare problems include changes in levels of aggression, changes in swimming position, lethargy, separation from the rest of the school and decreased avoidance of stimuli that would normally induce anxiety. Very sick fish become unresponsive to external stimuli and usually float at the surface or sink to the bottom of the tank. This can progress to a loss of equilibrium, with fish floating "belly up" as respiration rate slows. At this stage mortality usually occurs (see section on humane endpoints, below). A detailed list of clinical signs is given in the CCAC guidelines [58].

\subsubsection{Behavioral indications for pain and suffering}

Typical behavioral indicators of pain and suffering have been described for rainbow trout and zebrafish. In rainbow trout, clear signs of a noxious, potentially painful event are an increase in opercular beat rate from normal level of approximately 54 beats per minute to almost double (90e100 beats/min; [26]). This is a larger rise than a stress response (which is approximately 70 beats $/ \mathrm{min}$ ). Zebrafish also show an enhanced ventilation rate during nociception [30]. Trout perform anomalous behaviors such as rubbing of an affected area, loss of equilibrium [30] head shaking and scraping against objects [67]. There is also a 
dramatic decrease in activity in trout and zebrafish [30] but all of these changes show recovery from 3 to $6 \mathrm{~h}$ after the insult [33].

As in other experimental animals, observation and analysis of behavior is a valuable tool in research into pain and welfare in fish. Inspired by Morton and Griffiths [70], Midtlyng [71] proposed a set of behavioral, morphological, physiological and performance parameters to evaluate vaccination side-effects relevant to fish welfare. Detailed guidance on the evaluation of clinical (including behavioral) signs to help assess on the welfare of experimental fish has recently been published by the CCAC [58].

\subsubsection{Welfare assessment in practice}

Appendix B sets out an example of a welfare assessment system where numerical scores are assigned to different criteria; an alternative is the "binary" score sheet where behavioral signs are simply marked as present or absent.

A "team approach" is essential when designing and using any system for recognizing and recording adverse effects. This requires input from researchers, animal care staff and veterinarians, all of whom have different specialist knowledge and experience and can bring a different perspective. The team should also regularly review the assessment system and adapt it as necessary to ensure that it is flexible, effective, agreed on by all and usable by all. It is also important to maintain knowledge of new techniques for observing animals, such as behavioral recognition software, other remote monitoring systems or techniques for measuring metabolites in the water, so that these can be trialed and brought in whenever feasible. Appropriate training and Continuous Professional Development is essential, which may include specialist courses for those using and/or caring for fish. Staff levels should also be sufficient to ensure that there is enough time to monitor fish effectively.

For further guidance on welfare assessment see: $[58,64,71-73]$ and Appendix B.

Recommendations:

- It should be ensured that a comprehensive welfare assessment system has been set up. Subjective judgments about fish welfare and adverse effects are not always reliable in isolation.

- Welfare assessment should be tailored to the protocol and regularly reviewed, using a team approach.

- Information on welfare assessment criteria and systems should be shared with other fish users, e.g. in publications and at meetings.

- Information on new techniques for welfare assessment is essential; trial and use new methods whenever appropriate.

- It should be ensured that there is sufficient staff with adequate time to monitor the fish effectively.

\subsection{Humane endpoints}

Humane endpoints are an essential component of humane science in all fields and there is scope to implement humane endpoints in all vaccine studies, including those involving fish $[74,58]$. The Ph.Eur. general monograph on veterinary vaccines permits humane endpoints [47] and the use of effective welfare assessment systems, as outlined above, will facilitate their implementation. Considerable progress has been made with respect to humane endpoints in mammals and the workshop participants believe that fish deserve the same consideration.

However, there are some complicating factors in the case of vaccine testing and these need to be addressed. There is generally a regulatory requirement to demonstrate a degree of morbidity in vaccine 
tests, and it is necessary to be sure that animals are only killed if it is certain that they would have died as a result of the disease challenge within the given observation period. In other words, the clinical signs that determine the humane endpoint must be predictive of the required outcome of the test, e.g. mortality within a given period, because too many "false positives" can render a study invalid. In fish vaccine tests, even where endpoints have been defined, it can be difficult to identify fish that need to be euthanized in a tank containing large numbers.

In practice, sick fish may not be identified until they have reached a moribund condition, which has been described as a severely debilitated state that precedes imminent death. The moribund state is relatively easy to identify, but it is considered not desirable on welfare grounds because of the suffering that the animal will have experienced and may still be experiencing [75]. The general aim should be to reduce the number of fish that become moribund in vaccine studies by increasing the implementation of effective assessment systems and humane endpoints. However, in studies where challenge is by cohabitation it is necessary to leave the moribund fish in to maximize infectivity. In this case, there is a dilemma between allowing one fish to suffer vs. challenging all the fish by intraperitoneal injection.

Recommendations:

- The use of death as an endpoint should always be questioned, especially if it is not a regulatory requirement.

- Refinement of humane endpoints should be actively sought and the number of fish reaching a moribund state should be minimized within each study.

- More research is needed into the identification of humane endpoints for fish in vaccine studies.

- European Pharmacopoeia monographs for fish vaccines should specify typical clinical signs of the disease, include validated criteria for humane endpoints wherever available, and discourage death as an endpoint.

\subsection{Training}

All staff responsible for conducting procedures, monitoring fish and implementing humane endpoints or otherwise euthanizing fish must be both competent and empathetic. This requires not only that they are skilled in carrying out procedures, but also that they view fish as individuals, understand and accept that they can suffer, and appreciate why fish welfare is important. Training should thus include: the biology and behavior of the study species; recognizing and assessing both good welfare and signs of pain, suffering or distress; and competence in all procedures that may be carried out including euthanasia [76,77]. See also http://oslovet.veths.no/(click on "fish" and "education and training relating to fish in research" and Appendix B). In addition, the University of Prince Edward Island offers a complete training program for people who use fish for research, teaching and testing http://www.upei.ca/lifelonglearning/experimentalfish.

\section{Recommendation:}

- It should be ensured that the relevant staff is trained to be empathetic, as well as trained to be competent and knowledgeable about the species they are using and caring for.

\section{Summary of recommendations}

The participants in the workshop would like to encourage all stakeholders, manufacturers, regulators, researchers and animal welfare officers to follow-up the recommendations of the workshop, which can be summarized as follows: 


\subsection{Replacement}

- The use of antigen quantification methods for batch potency testing is being encouraged according to the opportunities given in the respective guidelines and monographs.

- Possibilities of applying the consistency approach to fish vaccine batch testing should be evaluated.

\subsection{Reduction}

- Fish vaccines produced in 3rd countries and imported to Europe should be only re-tested when scientifically justified and the current practice of routine re-testing should be omitted.

- Possibility of reducing the number of control fish in vaccination-challenge tests should be evaluated, since most of these unprotected fish are subjected to development of disease and associated distress.

- European regulators should harmonize their criteria for waiving the batch safety test. ${ }^{4}$

- The $\mathrm{VICH}$ should work toward harmonization of the requirements for the batch safety test for veterinary vaccines including fish vaccines. ${ }^{5}$

- Possibility of using single-dose protocols for batch safety testing should be discussed, which would allow combination with the batch potency test. ${ }^{4}$

\subsection{Refinement}

- Efforts should be undertaken to develop and validate antibody induction methods for batch potency testing, thus making vaccination-challenge tests obsolete. Funds should be made available for this.

- Death as an endpoint should be questioned whenever possible; humane endpoints should be applied and moribund fish be humanely killed.

- Less invasive administration techniques are to be preferred, e.g. waterborne exposure versus intraperitoneal injection, and the least invasive techniques for identification should be used.

- Welfare assessment criteria and systems for fish should be established and regularly reviewed in a team approach.

- Fish should be housed in species-appropriate social groups wherever protocols permit.

- Suitable environmental enrichment and other husbandry refinements should be sought and implemented.

- Staff should be trained in welfare assessment of fish, e.g. be able to detect signs of illness (e.g. change in skin color e especially darkened skin -, red gills, decreased opercular beat rate, eye color, physical injury, physical abnormalities and changes in oxygen demand) as well as behavioral changes (e.g. changes in levels of aggression and swimming position, lethargy, separation from the rest of the school and decreased avoidance of stimuli that would normally induce anxiety.

\footnotetext{
${ }^{4}$ Ongoing activities to remove the target animal safety test from. Ph.Eur. monographs (status 2010).

${ }^{5}$ Ongoing activities at $\mathrm{VICH}$ level on criteria for waiving the target animal safety test (status 2010).
} 


\section{Disclaimer}

The views expressed in this article are purely those of the author and may not in any circumstances be regarded as stating an official position of the European Commission.

\section{Acknowledgments}

The UK Animal Procedures Committee funded Penny Hawkins to participate in the workshop.

\section{Appendix. Supplementary data}

Supplementary data related to this article can be found online at doi:10.1016/j.biologicals.2011.02.001. Appendix A: The Regulatory Framework in Europe and North America for Fish Vaccines; and Appendix B: Animal Welfare Assessment of Fish - Example Score Sheet.

\section{References}

[1] Russell WMS, Burch RL. The principles of humane experimental technique. London: Methuen; 1959. Reprinted by UFAW, 1992.

[2] Norwegian Animal Research Authority. Annual report, http://www.fdu.no/fdu/; 2008 [accessed 18. 11. 10].

[3] Koppang EO, Fischer U, Satoh M, Jirillo E. Inflammation in fish as seen from a morphological point of view with special reference to the vascular compartment. Curr Pharm Des 2007;13:3649e55.

[4] Press CM, Evensen $\varnothing$. The morphology of the immune system in teleost fishes. Fish Shellfish Immunol 1999;9:309e18.

[5] Fletcher TC, Secombes CJ, Immunology of fish, In: Encyclopedia of Life Sciences. Chichester: John Wiley \& Sons e online library, http://www.els.net/doi:10.1038/npg.els.0000520, [accessed 18. 11. 10].

[6] Meseguer J, Lopez-Ruiz A, Garcia-Ayala A. Reticuloendothelial stroma of the head-kidney from the seawater teleost gilthead seabream (Sparus-Aurata L)--an ultrastructural and cytochemical study. Anat Rec 1995;241:303-9.

[7] Kaattari SL, Irwin MJ. Salmonid spleen and anterior kidney harbor populations of lymphocytes with different B-cell repertoires. Dev Comp Immunol 1985;9: 433-44.

[8] Reite OB. Comparative physiology of histamine. Physiol Rev 1972;52:778-819.

[9] Reite OB, Evensen $\varnothing$. Mast cells in the swim bladder of Atlantic salmon Salmo salar: histochemisty and responses to compound $48 / 80$ and formalin-inactivated Aeromonas salmonicida. Dis Aquat Org 1994;20:95-100.

[10] Magnadottir B. Innate immunity of fish (overview). Fish Shellfish Immunol 2006;20:137-51.

[11] Lukacs MF, Harstad H, Grimholt U, Beetz-Sargent M, Cooper GA, Reid L, et al. Genomic organization of duplicated major histocompatibility complex class I regions in Atlantic salmon (Salmo salar). BMC Genomics 2007;8:251. 
[12] Bengten E, Clem LW, Miller NW, Warr GW, Wilson M. Channel catfish immunoglobulins: repertoire and expression. Dev Comp Immunol 2006;30:77-92.

[13] Hansen JD, Landis ED, Phillips RB. Discovery of a unique Ig heavy-chain isotype (IgT) in rainbow trout: implications for a distinctive B cell developmental pathway in teleost fish. Proc Natl Acad Sci U S A 2005;102:6919-24.

[14] Hordvik I, Thevarajan J, Samdal I, Bastani N, Krossoy B. Molecular cloning and phylogenetic analysis of the Atlantic salmon immunoglobulin D gene. Scand J Immunol 1999;50:202-10.

[15] Li J, Barreda DR, Zhang YA, Boshra H, Gelman AE, Lapatra S, et al. B lymphocytes from early vertebrates have potent phagocytic and microbicidal abilities. Nat Immunol 2006;7:1116-24.

[16] Pilstrøm L. Adaptive immunity in teleosts: humoral immunity. Dev Biol 2005;121:23. Basel.

[17] Secombes CJ, Bird S, Zou J. Adaptive immunity in teleosts: cellular immunity. Dev Biol 2005;121:25-32. Basel.

[18] Somamoto $T$, Okamoto $N$, Nakanishi $T$, Ototake $M$, Nakao M. In vitro generation of viral-antigen dependent cytotoxic T-cells from ginbuna crucian carp, Carassius auratus langsdorfii. Virology 2009;389:26-33.

[19] Utke K, Bergmann S, Lorenzen N, Köllner B, Ototake M, Fischer U. Cell-mediated cytotoxicity in rainbow trout, Oncorhynchus mykiss, infected with viral haemorrhagic septicaemia virus. Fish Shellfish Immunol 2007;22:182-96.

[20] Boudinot P, Boubekeur S. A.Benmansour, Rhabdovirus infection induces public and private $T$ cell responses in teleost fish. J Immunol 2001;167:6202-9.

[21] Sneddon LU. Ethics and welfare; pain perception in fish. Bull Eur Assoc Fish Path 2006;26:6-10.

[22] International Association for the Study of Pain (IASP). Pain terms: a list with definitions and notes on usage. Pain 1979;6:249-52.

[23] Sneddon LU. Anatomical and electrophysiological analysis of the trigeminal nerve in the rainbow trout Onchorhynchus mykiss. Neurosci Lett 2002; 39:67-171.

[24] Sneddon LU. Trigeminal somatosensory innervation of the head of the rainbow trout with particular reference to nociception. Brain Res 2003;972:44-52.

[25] Sneddon LU. Evolution of nociception in vertebrates: comparative analysis of lower vertebrates. Brain Res Rev 2004;46:123-30.

[26] Sneddon LU, Braithwaite VA, Gentle MJ. Do fish have nociceptors? Evidence for the evolution of a vertebrate sensory system. Proc Roy Soc Lond B 2003; 270:1115-21.

[27] Ashley PJ, Sneddon LU, McCrohan CR. Properties of corneal receptors in a teleost fish. Neurosci Lett 2006;410:165-8.

[28] Ashley PJ, Sneddon LU, McCrohan CR. Nociception in fish: stimulus-response properties of receptors on the head of trout Oncorhynchus mykiss. Brain Res 2007;1166:47-54. 
[29] Dunlop R, Laming P. Mechanoreceptive and nociceptive responses in the central nervous system of goldfish (Carassius auratus) and trout (Oncorhynchus mykiss). J Pain 2006;6:551-68.

[30] Reilly SC, Quinn JP, Cossins AR, Sneddon LU. Behavioural analysis of a nociceptive event in fish: comparisons between three species demonstrate specific responses. Appl Anim Behav Sci 2008;114:248-59.

[31] Sneddon LU. The evidence for pain in fish; the use of morphine as an analgesic. Appl Anim Behav Sci 2003;83:153-62.

[32] Ehrensing RH, Michell GF, Kastin AJ. Similar antagonism of morphine analgesia by MIF-1 and naxolone in Carassius auratus. Pharm Biochem Behav 1982;17:757-61.

[33] Sneddon LU, Braithwaite VA, Gentle MJ. Novel object test, Examining nociception and fear in the rainbow trout. J Pain 2003;4:431-40.

[34] Ashley PJ, Ringrose S, Edwards KL, Wallington E, McCrohan CR, Sneddon LU. Effect of noxious stimulation upon anti-predator responses and dominance status in rainbow trout. Anim Behav 2009;77:403-10.

[35] Clayton NS, Dickinson A. What, where and when: episodic-like memory during cache recovery by scrub jays. Nature 1998;395:272-4.

[36] Morris RG. Episodic-like memory in animals: psychological criteria, neural mechanisms and the value of episodic-like tasks to investigate animal models of neurodegenerative disease. Philos Trans R Soc Lond B Biol Sci 2001;356:1453-65.

[37] Reiss D, Marino L. Mirror self-recognition in the bottlenose dolphin: a case of cognitive convergence. Proc Natl Acad Sci U S A 2001;98:5937-42.

[38] Smith J, Shields W, Washburn D. The comparative psychology of uncertainty monitoring and metacognition. Behav Brain Sci 2003;26:317-73.

[39] Wynne C. Do animals think? Princeton, NJ: Princeton University Press; 2004. [40] Hendriksen C, Arciniega JL, Bruckner L, Chevalier M, Coppens E, Descamps J, et al. The consistency approach for the quality control of vaccines. Biologicals 2008;36:73-7.

[41] United States Department of Agriculture. Veterinary services memorandum 800.90. Guidelines for veterinary biological relative potency assays and reference preparations based on ELISA antigen quantification,

http://www.aphis.usda.gov/animal_health/vet_biologics/publications/memo_800_90.pdf; 1998 [accessed 18. 11. 10].

[42] Council of Europe. 5.3. Statistical analysis of results of biological assays and tests. 6th ed. European Pharmacopoeia; 2008.

[43] VICH. VICH Topic GL2: validation of analytical procedures: methodology (CVMP/VICH/591/98), http://www.vichsec.org/pdf/gl02_st7.pdf;1998 [accessed 18. 11. 10].

[44] European Commission. Directive 2001/82/EC of the European Parliament and of the Council of 6 November 2001 on the Community code relating to veterinary medicinal products. Off $\mathrm{J}$ Eur Union 2001;L311:1-66. 
[45] European Commission. The Rules Governing Medicinal Products in the European Union-EudraLex Volume 7B: Specific requirements for the production and control of live and inactivated vaccines intended for fish-7blm9a http://ec.europa.eu/health/files/eudralex/vol-7/b/7blm9a_en.pdf [accessed 18. 11. 10].

[46] VICH. VICH GL 44-Target animal safety for veterinary live and inactivated vaccines (EMEA/CVMP/VICH/359665/2005), http://www.vichsec.org/pdf/0708/GL44-st7.doc; 2008 [accessed 18. 11. 10].

[47] Council of Europe. Vaccines for veterinary use, monograph no 62. 6th ed. European Pharmacopoeia; 2008.

[48] Ema. Cvmp-position paper on data requirements for removing the target animal batch safety test for immunological veterinary medicinal products in the EU (EMEA/CVMP/865/03/Final), http://www.ema.europa.eu/docs/en_GB/document_library/Scientific_guideline/2009/10/WC5000045 91.pdf 2004 [accessed 18. 11. 10].

[49] Castle P. Replacement, reduction, refinement (3rs)-animal welfare progress in european pharmacopoeia monographs. Pharmeuropa 2007;19:430-40.

[50] Midtlyng PJ. Critical assessment of regulatory standards and tests for fish vaccines. In: Midtlyng PJ, editor. Progress in fish Vaccinology. Dev Biol, vol. 121; 2005. p. 219-26. Basel.

[51] Jarp J, Tverdal A. Statistical aspects of fish vaccination trials. In: Gudding R, Lillehaug A, Midtlyng PJ, Brown F, editors. Fish Vaccinology. Dev Biol Stand, vol. 90; 1997. p. 311-20.

[52] Nordmo R. Stengths and weaknesses of different challenge methods. In: Gudding R, Lillehaug A, Midtlyng PJ, Brown F, editors. Fish Vaccinology. Dev Biol Stand, vol. 90; 1997. p. 303-9.

[53] Hendriksen CFM, Garthoff B, Aggerbeck H, Bruckner L, Castle P, Cussler K, et al. Alternatives to animal testing in the quality control of immunobiologicals: current status and future prospects. The report and recommendations of ECVAM workshop 4. ATLA 22; 1994. 420-434.

[54] Grimholt U, Getahun A, Hermsen T, Stet RJ. The major histocompatibility complex class II alpha chain in salmonid fishes. Dev Comp Immunol 2000;24:751-63.

[55] Grimholt U, Larsen S, Nordmo R, Midtlyng P, Kjøglum S, Storset A, et al. MHC polymorphism and disease resistance in Atlantic salmon (Salmo salar); facing pathogens with single expressed major histocompatibility class I and class II loci. Immunogenetics 2003;55:210-9.

[56] Wiegertjes GF. Immunogenetics of disease resistance in fish. Doctoral thesis, Wageningen Agricultural University; 1995.

[57] Buchanan-Smith $H$, Rennie AE, Vitale A, Pollo $S$, Prescott $M J$, Morton DB. Harmonising the definition of refinement. Anim Welf 2005;14:379-84.

[58] Canadian Council on Animal Care. Guidelines on: the care and use of fish in research, teaching and testing. Ottawa: Canadian Council on Animal Care, http://www.ccac.ca; 2005 [accessed 18. 11. 10].

[59] Ortuno J, Esteban MA, Meseguer J. Effects of four anaesthetics on the innate immune response of gilthead seabream (Sparus aurata L.). Fish Shellfish Immunol 2002;12:49-59. 
[60] Koppang EO, Bjerkäs I, Haugarvoll E, Chan EKL, Szabo NJ, Ono N, et al. Vaccination-induced systemic autoimmunity in farmed atlantic salmon. J Immunol 2008;181:4807-14.

[61] Morton DB, Jennings M, Buckwell A, Ewbank R, Godfrey C, Holgate B, et al, Joint Working Group on Refinement. Refining procedures for the administration of substances. Lab Anim 2001;35:1-41. Report of the BVAAWF/FRAME/RSPCA/UFAW Joint Working Group on Refinement. British Veterinary Association Animal Welfare Foundation/Fund for the Replacement of Animals in Medical Experiments/Royal Society for the Prevention of Cruelty to Animals/Universities Federation for Animal Welfare.

[62] European Commission. Commission recommendation of 18 June 2007 on guidelines for the accommodation and care of animals used for experimental and other scientific purposes (2007/526/EC). Off J Eur Union 2007; L97:1-89.

[63] Animal Procedures Committee. Supplementary review of schedule 1 of the animals (scientific procedures) act 1986: appropriate methods of humane killing for fish. London: APC, http://apc.homeoffice.gov.uk/reference/apc_supplementary_review_schedule_1.pdf; 2009 [accessed 18. 11. 10].

[64] Johansen R, Needham JR, Colquhoun DJ, Poppe TT, Smith AJ. Guidelines for health and welfare monitoring of fish used in research. Lab Anim 2006;40:323-40.

[65] Berg A, Rödseth OM, Tangeraas A, Hansen T. Time of vaccination influences development of adhesions, growth and spinal deformities in Atlantic salmon Salmo salar. Dis Aquat Organ 2006;69:239-48.

[66] Handley JW. Environmental enrichment for fish used in regulatory toxicology studies. Anim Technol 2001;52:227-31.

[67] Animal Procedures Committee. Unpublished data: results of a survey of UK fish users; 2008.

[68] Poole T, English P, editors. The UFAW handbook on the care and management of laboratory animals volume 2: amphibious and aquatic vertebrates and advanced invertebrates. 7 th ed. Oxford: Blackwell Science; 1999.

[69] Ostrander G, Bullock G, Bunton T. The laboratory fish; 2000. Amsterdam. [70] Morton DB, Griffiths PHM. Guidelines on the recognition of pain, distress and discomfort in experimental animals and an hypothesis for assessment. Vet Rec 1985;116:431-6.

[71] Midtlyng PJ. Vaccinated fish welfare: protection vs. side-effects. In: Gudding R, Lillehaug A, Midtlyng PJ, Brown F, editors. Fish Vaccinology. Dev Biol Stand, vol. 90; 1997. p. 371-9.

[72] Morton DB. A scheme for the recognition and assessment of adverse effects in animals. In: van Zutphen LFM, Balls M, editors. Animal alternatives, welfare and ethics. Amsterdam: Elsevier; 1997. p. 235-40.

[73] Hawkins P. Recognizing and assessing pain, suffering and distress in laboratory animals: a survey of current practice in the UK with recommendations. Lab Anim 2002;36:378-95.

[74] Cussler K, Morton DB, Hendriksen CFM. Humane endpoints in vaccine research and quality control. In: Hendriksen CFM, Morton DB, editors. Humane endpoints in animal experiments for biomedical research. London: Royal Society of Medicine Press; 1999. p. 95-101. 
[75] Toth LA. Defining the moribund condition as an experimental endpoint for animal research. ILAR J 2000;41:72-9.

[76] Felasa. FELASA recommendations on the education and training of persons working with laboratory animals: categories A and C. Lab Anim 1995;29:121-31.

[77] Felasa. FELASA recommendations on the education and training of persons carrying out animal experiments (Category B). Lab Anim 2000;34:229-35. 